\title{
Stage III Vaginal Cancer AJCC v6 and v7
}

National Cancer Institute

\section{Source}

National Cancer Institute. Stage III Vaginal Cancer A/CC v6 and v7. NCI Thesaurus. Code C7858.

Stage III includes: (T1-3, N1, M0) and (T3, NO, M0). T1: T umor confined to vag ina. T2: Tumor invades paravaginal tissues but not to pelvic wall. T3: Tumor extends to pelvic wall. N0: No regional lymph node metastasis. N1: Pelvic or inguinal lymph node metastasis. M0: No distant metastasis. (AJCC 6th and 7th eds.) 